\title{
Cranioplasty using custom-made hydroxyapatite versus titanium: a randomized clinical trial
}

\author{
Dirk Lindner, MD, ${ }^{1}$ Kathrin Schlothofer-Schumann, ${ }^{1}$ Bodo-Christian Kern, MD, ${ }^{2}$ \\ Omeima Marx, MD, ${ }^{3}$ Andrea Müns, ${ }^{1}$ and Jürgen Meixensberger, MD ${ }^{1}$
}

\begin{abstract}
1Department of Neurosurgery, Leipzig University, Leipzig; ${ }^{2}$ Department of Neurosurgery, BG Clinic Bergmannstrost, Halle; and ${ }^{3}$ Department of Neurosurgery, Nuremberg Hospital, Nuremberg, Germany
\end{abstract}

\begin{abstract}
OBJECTIVE Cranioplasty is routinely performed in neurosurgery. One of its underestimated problems is the high postoperative complication rate of up to $40 \%$. Due to the lack of good prospective studies and the small number of patients (5-20 each year) who receive alloplastic materials, decisions in favor or against a certain material are based on subjective empirical or economic reasons. The main goal of this study - the first prospective, randomized multicenter study in Germany-of custom-made titanium and hydroxyapatite (HA) implants was to compare local and systemic infections related to the implant within the first 6 months after implantation. Secondary objectives included comparing the reoperation rate, the complication rate, clinical and neurological outcomes, and health-related quality of life.
\end{abstract}

METHODS The study included patient screening and randomization at 6 to 8 weeks before operation; pre-, intra-, and postoperative documentation until discharge; and postoperative follow-ups after 1 and 6 months. Approval for the study was obtained from the local ethics committee.

RESULTS A total of 52 patients were included in the study. The rate of local implant-associated wound infection in the HA group was 2 of $26(7.7 \%)$ patients and 5 of $24(20.8 \%)$ patients in the titanium group ( $p=0.407)$. Systemic inflammation within 6 months after operation affected none of the patients in the HA group and 4 of $24(37.5 \%)$ patients in the titanium group ( $p=0.107$ ). In both groups, 7 patients required reoperation after the 6 -month follow-up ( $26.9 \%$ of the HA group and $29.2 \%$ of the titanium group; not significant). Reoperation with an explantation was necessary in 3 patients in each group (11.5\% of the HA group and $12.5 \%$ of the titanium group; not significant). The results demonstrated a significantly higher number of epidural hematomas in the HA group in comparison with none in the titanium group. Altogether, 46 adverse events were found in 27 patients (54\%). An improvement in the neurological outcome after 6 months was experienced by $43 \%$ of the patients in the HA group and $26.3 \%$ of the patients in the titanium group $(p=0.709)$.

CONCLUSIONS The study emphasizes that cranioplasty is a high-risk intervention. In comparison with titanium, HA shows benefits in terms of the infection rate and the neurological outcome, but at the same time has a higher postoperative risk for epidural hematoma. Depending on the individual conditions, both materials have their place in future cranioplasty therapies.

Clinical trial registration no.: NCT00923793 (clinicaltrials.gov).

http://thejns.org/doi/abs/10.3171/2015.10.JNS151245

KEY WORDS cranioplasty; titanium; hydroxyapatite; prospective study

$\mathrm{N}$ OWADAYS, cranioplasty is used worldwide in all neurosurgical departments. The success of implantation depends on a variety of factors, such as the size and location of cranioplasty, as well as the patient's condition, the material used, and the implantation time. ${ }^{1,9,30}$ A range of studies has targeted these parameters retrospectively. ${ }^{3,19}$ All studies have shown that complications occur much more often than generally assumed. In the worst cases, the consequences range from infections and lengthy hospitalization to implant loss and even mortality. ${ }^{5,27}$ On the other hand, younger residents often perform this surgery as a learning experience. The impact of the choice of the surgeon on the success of intervention can only be evaluated in a prospective study.

The idea of collaboration between maxillofacial and neurosurgical specialists, as practiced for example in the Benelux countries, is an interesting approach, especially when it comes to difficult wound conditions or repetitive

ABBREVIATIONS AE = adverse event; CCT = cranial computed tomography; GCS = Glasgow Coma Scale; HA = hydroxyapatite; KPS = Karnofsky Performance Scale; mGOS = modified Glasgow Outcome Scale; SAE = significant AE; SF-36 = 36-Item Short Form Health Survey.

SUBMITTED May 29, 2015. ACCEPTED October 26, 2015.

INCLUDE WHEN CITING Published online February 26, 2016; DOI: 10.3171/2015.10.JNS151245. 
interventions on the same patient. ${ }^{11}$ Unfortunately, such collaborations are still rare in Germany. The impact on the wound-healing process can also only be evaluated by means of a prospective survey. ${ }^{21}$

Intense debate surrounds the choice of the most suitable material for each patient. There is reasonable assurance that reimplantation of the patient's bone flap leads to aseptic bone necrosis in up to $25 \%$ of cases and may necessitate replacement with an alloplastic material. Many other studies have also investigated the impact of bone flap storage, as well as the impact of the length of time until reimplantation. ${ }^{15,20,31}$ Due to the high costs of bone banks, in Italy for example, there is a trend toward the almost exclusive use of alloplastic materials. ${ }^{24}$ Although some German hospitals are now following this trend, legal assessment of this approach is still pending.

The manufacturers of appropriate materials emphasize their excellent compatibility, high cosmetic properties, and simple use. ${ }^{17,23}$ Due to the lack of good prospective studies and the low number of patients (5-20 each year) who receive alloplastic materials, decisions for or against a certain material are based on subjective empirical or economic reasons. However, this manner of decision making is no longer acceptable given the high costs of each cranioplasty and led to the foundation of the German and UK Cranial Reconstruction Registry in $2014 .^{10}$ The aim is to gain valid data about each cranioplasty.

This study-the first prospective, randomized multicenter study conducted in Germany- of 2 common materials was planned back in 2009. The materials compared are porous hydroxyapatite [HA] (CustomBone [Finceramica]) and titanium (DMD Dortmund). Both manufacturers produce implants on the basis of individual CT data sets and a virtual computer-aided design construction process. ${ }^{6,24} \mathrm{~A}$ randomized multicenter study was favored in order to gain data from a higher number of patients in a shorter time period.

\section{Methods \\ Hypothesis}

The primary objective was to compare local implantassociated infections and/or systemic infections in both study arms within the first 6 months after implantation. An infection rate of $15 \%{ }^{25}$ was assumed for titanium in comparison with $2 \%$ for HA. ${ }^{8}$

Further secondary objectives included 1) comparison of the rate of reoperation with and without explantation; 2) comparison of the patients' neurological and clinical status; 3) comparison of surgery time, anesthesia time, complications, and CT scans; 4) comparison of health-related quality of life on the 36-Item Short Form Health Survey (SF-36) after cranioplasty; 5) comparison of modified Glasgow Outcome Scale (mGOS) score, ${ }^{18}$ Karnofsky Performance Scale (KPS) score, and overall impression; 6) group comparisons based on the cosmetic results, duration of hospital stay, and treatment costs; and 7) comparison of temperature sensitivity (heating and cooling) at 6 months after implantation.

It was hoped that the study (clinical trial registration no. NCT00923793 [clinicaltrials.gov]) would indicate the types of cases for which each material is better suited, as well as the risks and benefits that may arise as a result of cranioplasty.

\section{Materials \\ Titanium}

Due to its excellent biocompatibility, fitting accuracy, and stability, pure titanium has been used in Germany to make implants for cranioplasty for more than 10 years. Every implant is custom-made preoperatively based on CT data. Titanium implants are delivered nonsterile and need to be cleaned and sterilized. After cleaning, steam sterilization has to be performed at $134^{\circ} \mathrm{C}$ for at least 4 minutes. ${ }^{6}$

\section{CustomBone}

CustomBone implants are sterilized using gamma radiation (2.5 Mrad). They are designed only for individual use. The biomimetic approach is followed by the use of HA, which makes up 70\% of human bone. The special feature of the CustomBone implant made of synthetic HA is the computer-aided design/manufacturing process based on a CT data set of the patient. This material is intended to support bone regeneration, colonize osteogenic cells, be aesthetically precise, and show behaviors similar to that of natural bone material at 12 weeks postoperatively. Moreover, the implant is designed to provide highly accurate geometry and fit. The required biological interaction has already been confirmed in animal studies. The implants can be stored at room temperature. ${ }^{22}$

\section{Participation in the Study Inclusion Criteria}

The following inclusion criteria were used in this study: 1) cranial defect with no possibility of replacement with the patient's own bone flap; 2) defect size $\geq 16 \mathrm{~cm}^{2}$; 3) age $>18$ years; and 4) written declaration of consent.

\section{Exclusion Criteria}

The following exclusion criteria were used in this study: 1) active tumor; 2) American Society of Anesthesiologists Class IV (anesthesiology classification for narcosis); 3) allergic disposition to ceramics or titanium; 4) concomitant participation in other clinical trials; 5) pregnant or nursing women; 6) expected low compliance; 7) HIV-positive diagnosis; and 8) active drug abuse.

\section{Course of the Study}

At 6 to 8 weeks before surgery, the patient was enrolled in the study, underwent a clinical examination, provided informed consent, randomization was performed, bone that was $1-\mathrm{mm}$ thick on 3D cranial computed tomography (CCT) was confirmed, and the implant was ordered. Preoperative status was evaluated 1 to 2 days before surgery using clinical and neurological examinations (including Glasgow Coma Scale [GCS], mGOS, and KPS scores), local defects were assessed, patients were excluded due to wound infection, laboratory parameters were assessed, and the SF-36 questionnaire was administered. Intraop- 
erative status was evaluated according to the implantation procedure, duration of surgery and anesthesia, and complications/adverse events (AEs). Postoperative status was evaluated in terms of the complications/AE/significant AEs (SAEs), CCT findings, and implant fit. Discharge occurred at about 4 to 8 days postoperatively, and the patient was evaluated using clinical and neurological examination (including GCS, mGOS, and KPS scores) and complications/AE/SAE, duration of hospital stay, cosmetic results, and scarring were assessed. At 1 month postoperatively, clinical and neurological examination (including GCS, mGOS, and KPS scores) and assessments of complications/AEs/SAEs, duration of hospital stay, cosmetic results, scarring, temperature sensitivity, and SF-36 were performed. At 6 months postoperatively, clinical and neurological examination (including GCS, mGOS, and KPS scores) and assessments of complications/AE/SAEs, duration of hospital stay, cosmetic results, scarring, temperature sensitivity, and SF-36 were performed.

Approval was obtained from the local ethics committee for this intention-to-treat study. The manufacturers Finceramica and Codman \& Shurtleff Inc. shared the funding equally.

\section{Statistical Analysis}

Sample size and power calculations were based on a comparison of the postoperative infection rates when Fisher's exact test was used. A randomization ratio of 1:1 and sample size of 77 patients per arm would ensure a significance level of $5 \%$ and a power of $80 \%$ to test the null hypothesis of $15 \%$ postoperative infections in both treatment arms against the alternative of a $13 \%$ lower rate of infections when HA implants ${ }^{25}$ were used. Estimations of power with respect to the major secondary end points of reoperation and explantation are based on literature references, which report $10 \%$ to $13 \%$ for the former and $8 \%$ for the latter when titanium implants were used ${ }^{8}$ in comparison with $0.5 \%$ for both reoperation and explantation for HA. ${ }^{25}$ For a sample size of 77 patients per arm, this results in an expected power of $70 \%$ for these end points. Because of the framework requirements of the study's performance, the aim was to include 150 patients over a total period of 2 to 3 years.

To generate the random allocation sequence, the dynamic Pocock minimization algorithm was used. The stratification characteristics were the size of the defect ( $\leq$ $100 \mathrm{~cm}^{2}$ vs $>100 \mathrm{~cm}^{2}$ ) and the capacity of the patient to give consent or not, which were weighted differently.

\section{Recruitment}

Although the original plan was to have 6 to 8 clinical centers participate in the study for statistical reasons, only 3 clinics signed up as study partners. One problem was the comprehensive recruitment of all potential patients, and another problem was the obvious favoring of one cranioplasty material over the other by individual clinics. Accordingly, recruitment ceased in January 2013, and the last follow-up evaluation took place in early August 2013. Statistical analysis, randomization, and support during the study were carried out by the Clinical Trial Centre Leipzig, an independent institute.

\section{Results}

A total of 53 patients were included in the study, although 1 patient dropped out following randomization after opting for the other material for subjective reasons. Descriptive information about the patients at the start of the study is contained in Table 1.

In greater than $90 \%$ of the patients, cranioplasty for hemispherical or frontotemporal defects was performed. Only 5 patients ( 2 in the HA group and 3 in the titanium group) showed a bifrontal skull defect. One patient died of pulmonary embolism between joining the study and undergoing surgery, and another broke off contact before cranioplasty. Altogether 50 patients (26 patients in the HA group and 24 patients in the titanium group) were analyzed regarding the primary and secondary objectives.

\section{Primary Objective}

The occurrence of local, implant-associated wound infection within 6 months after operation affected 2 of 26 (7.7\%) patients in the HA group and 5 of 24 (20.8\%) patients in the titanium group (Fisher's exact test, $\mathrm{p}=0.407$ ). All of these patients also underwent a reoperation. Four of 24 patients in the titanium group suffered solely systemic infection, which was not related to the implant, in comparison with none in the HA group (Fisher's exact test, $\mathrm{p}=0.107)$. Considering local and systemic infection together, the HA group had 2 of $26(7.7 \%)$ patients in comparison with 9 of $24(37.5 \%)$ patients in the titanium group (Fisher's exact test, $\mathrm{p}=0.031$ ), which is a significant result (Table 2). During follow-up, 2 patients in the HA group and 6 patients in the titanium group underwent additional antibiotic treatment (Fisher's exact test, $\mathrm{p}=0.238$ ).

\section{Secondary Objectives}

Comparison of the Rate of Reoperations With and Without Explantation

In both groups, 7 patients had to undergo reoperation during the 6-month follow-up (26.9\% in the HA group and $29.2 \%$ in the titanium group; Fisher's exact test, $\mathrm{p}=1.0$ ).

The reasons for reoperation in the HA group were infection $(\mathrm{n}=2)$ and epidural hematoma with mass effect (n $=3 ; 1$ patient was immediately reoperated on because of a newly detected cerebellar angioma with cerebellar and epidural hematoma). In 2 patients, small epidural hematomas were associated with the presence of a shunt system, and reoperation stopped the shunt function. In the titanium group, 5 patients underwent reoperation because of infection and 2 patients because of other cancer diseases.

Reoperation with explantation proved necessary in 3 patients in each group (11.5\% in the HA group and $12.5 \%$ in the titanium group; Fisher's exact test, $\mathrm{p}=1.0$ ). In the HA group, the reasons were 2 cases of infection and 1 epidural hematoma; in the titanium group, all 3 patients underwent reoperation because of infection.

\section{Comparison of the Patients' Pre- and Postoperative Neurological and Clinical Status}

The numbers of patients with neurological disorders were relatively high in both groups (Table 1). Postoperatively and in the follow-up period, the numbers of patients 
TABLE 1. Preoperative patient characteristics in the HA and titanium groups*

\begin{tabular}{|c|c|c|}
\hline Variable & Hydroxyapatite & Titanium \\
\hline No. of patients & 26 & 26 \\
\hline Implant size, $>100 \mathrm{~cm}^{2} /<100 \mathrm{~cm}^{2}$ & $13 / 13$ & $13 / 13$ \\
\hline Median cranioplasty size $\left(\mathrm{cm}^{2}\right)$ & 116.9 & 126.2 \\
\hline Legal representative $†$ & $30.80 \%$ & $26.90 \%$ \\
\hline Male/female, \% & $61.5 / 38.5$ & $57.7 / 42.3$ \\
\hline Mean age \pm SD (at study recruitment) & $48.6 \pm 15.4$ & $53.1 \pm 14.1$ \\
\hline Body mass index & $26.7 \pm 4.9$ & $26.2 \pm 5.4$ \\
\hline Internal diseases $\ddagger$ & $65.40 \%$ & $62.50 \%$ \\
\hline Neurological disorders & $80.80 \%$ & $83.30 \%$ \\
\hline \multicolumn{3}{|l|}{ Reason for primary op } \\
\hline Subdural hematoma & 9 & 6 \\
\hline Traumatic decompression & 5 & 7 \\
\hline Aneurysm op & 3 & 3 \\
\hline Tumor op & 4 & 9 \\
\hline Infarct decompression & 2 & 1 \\
\hline Postop CSF leakage & 2 & \\
\hline Cerebral hematoma & 1 & \\
\hline \multicolumn{3}{|l|}{ Reason for 2nd op§ } \\
\hline Primary op & 3 & 5 \\
\hline Infection & 14 & 17 \\
\hline Aseptic osteonecrosis & 9 & 4 \\
\hline \multicolumn{3}{|l|}{ mGOS score at study recruitment } \\
\hline Group 1 (good recovery) & 10 & 13 \\
\hline Group 2 (moderate disability) & 4 & 2 \\
\hline Group 3 (severe disability) & 12 & 9 \\
\hline \multicolumn{3}{|l|}{ KPS score } \\
\hline Mean \pm SD & $68.5 \pm 22.2 \%$ & $74.6 \pm 25.5 \%$ \\
\hline Range & $20-100 \%$ & $30-100 \%$ \\
\hline \multicolumn{3}{|l|}{ Preop preparation } \\
\hline Trendelenburg positioningף & 5 & 3 \\
\hline Lumbar drainage $^{* *}$ & 1 & 1 \\
\hline Preop antibiotics & 0 & 1 \\
\hline Local decubitus & 5 & 7 \\
\hline
\end{tabular}

* Data are presented as the number of patients unless otherwise indicated. $\dagger$ Percentage of patients who were unable to provide informed consent and legal representation was necessary.

$\ddagger$ Arteriosclerosis, diabetes, high blood pressure, lipometabolic disorder, and active cancer.

$\S$ Some operations included decompression (e.g., multiple fractures without the possibility of reconstruction of the natural bone).

If Preparation for patients with a deeply sunken brain surface in the skull defect.

** Preparation for patients with strongly prominent brain surface in the skull defect.

were nearly similar in both groups, and after 6 months $90.5 \%$ of the patients in the HA group and $80 \%$ in the titanium group suffered neurological disorders (chi-square test, $\mathrm{p}=0.406$ ).

The numbers of patients with internal diseases were also relatively high in both groups (Table 1). Postoperatively and in the follow-up period, the numbers of patients
TABLE 2. Evaluation of the primary hypothesis regarding infection in the HA and titanium groups

\begin{tabular}{llll}
\hline \multirow{2}{*}{ Variable } & \multicolumn{2}{c}{ No. of Patients (\%) } & \\
\cline { 2 - 3 } & HA & Titanium & p Value* \\
\hline No. of patients & 26 & 24 & \\
\hline No. w/ infections & $2(7.7)$ & $9(37.5)$ & $0.031 \dagger$ \\
\hline \multicolumn{1}{c}{ Implant-associated local infection } & $2(7.7)$ & $5(20.8)$ & 0.407 \\
\hline \multicolumn{1}{c}{ Systemic infection } & 0 & $4(16.7)$ & 0.107 \\
\hline Additional antibiotic treatment & $2(7.7)$ & $6(25)$ & 0.238 \\
\hline * Fisher's
\end{tabular}

* Fisher's exact test.

$\dagger$ Statistically significant.

with internal diseases did not change (chi-square test: $\mathrm{p}$ $=1.0$ ).

The change in neurological outcome was also measured and compared between both groups before and after implantation (Table 3).

\section{Comparison of Surgery Time, Anesthesia Time, Intraoperative Complications, and CT Scan}

Intraoperative parameters such as surgery time, anesthesia time, and intraoperative events, as well as postoperative CT scan results and quality analysis, are listed in Table 4 . The main findings are the significantly shorter operation time in the titanium group versus the HA group, the significantly improved evaluation of CT scans in the HA group, and the significantly higher number of epidural hematomas in the HA group in comparison with the titanium group.

\section{Comparison of the Health-Related Quality of Life After Cranioplasty}

The SF-36 questionnaire was used to evaluate the patients' subjective quality of life. Response rates were relatively high, with nearly $80 \%$ at the beginning, nearly $70 \%$ at 1 month postoperatively, and $60 \%$ at 6 months postoperatively.

The questionnaire consisted of 8 sets of physical and mental tests. No significant changes were measured by any of them, with the exception of the total psychological score (Mann-Whitney U-test, $p=0.035$; OR $-9.4 ; 95 \% \mathrm{CI}$ -17.9 to -0.41 ) in favor of titanium. Moreover, in the first section (patient's assessment of the change in health), only $58 \%$ of the patients in the titanium group felt the same or better in comparison with 1 year beforehand, whereas in the HA group $79 \%$ felt the same or better in comparison with 1 year beforehand. At 1 month postoperatively, the difference decreased $(81.8 \%$ in the HA group vs $73.7 \%$ in the titanium group), and after 6 months there was no difference between the 2 groups ( $94.1 \%$ in the HA group vs $94.1 \%$ in the titanium group). Patients in both groups were more satisfied at the end of the study than at the beginning, a greater increase in satisfaction being noted in the titanium group.

\section{Comparison of mGOS and KPS Scores and Overall Impression}

The mGOS defines 5 groups. Table 5 shows a slight nonsignificant improvement over time, but no significant differences between groups. With an mGOS score of 5 , 
TABLE 3. Changes in neurological disorders after operation in the $\mathrm{HA}$ and titanium groups

\begin{tabular}{cccc}
\hline $\begin{array}{c}\text { Neurological Outcome } \\
\text { Before vs After Surgery }\end{array}$ & Discharge & $\begin{array}{c}\text { 1-Mo } \\
\text { Follow-Up }\end{array}$ & $\begin{array}{c}\text { 6-Mo } \\
\text { Follow-Up }\end{array}$ \\
\hline HA group & & & \\
\hline Improved & 1 & 5 & $9(43 \%)$ \\
\hline Unchanged & 22 & 16 & 12 \\
\hline Impaired & 1 & 1 & 0 \\
\hline Total & 24 & 22 & 21 \\
\hline Titanium group & & & \\
\hline Improved & & 3 & $5(26.3 \%)$ \\
\hline Unchanged & 22 & 18 & 13 \\
\hline Impaired & 1 & 2 & 1 \\
\hline Total & 23 & 23 & 19 \\
\hline Chi-square test & $\mathrm{p}=0.554$ & $\mathrm{p}=0.927$ & $\mathrm{p}=0.709$ \\
\hline
\end{tabular}

1 patient from the titanium group died before implantation was started (pulmonary embolism). One patient died after implantation because of an unidentified angioma with fatal hematoma, and a second patient died postoperatively of an unidentified middle and posterior cerebral artery infarction. In the long-term follow-up, one patient died of newly detected lung cancer and another of the consequences of sepsis.

The KPS score was nearly the same in both groups preoperatively $(68.5 \% \pm 22.2 \%$ in the HA group vs $74.6 \% \pm$ $25.5 \%$ in the titanium group). The values were unchanged upon discharge and at the 1-month follow-up and had clearly improved by the 6-month follow-up $(75 \% \pm 23.9 \%$ vs $78 \% \pm 25.6 \%$ ), with the difference between the groups being insignificant.

The overall impression of the implant procedure and the follow-up was measured using 4 subjective criteria (very good, good, satisfactory, and unsatisfactory). The criteria had to be rated by the doctor and the patient independently. The results are not surprising, with the patients' overall impression of the implantation and the follow-up being slightly more pessimistic than those of the doctor. No significant differences were found between the 2 groups at the different study times (more than $90 \%$ of the patients always had a positive impression in both groups).

\section{Group Comparison Based on the Cosmetic Results, Hospital Stays, and Treatment Costs}

Upon discharge, wound control revealed signs of skin irritation without consequences in 5 patients $(20 \%)$ in the HA group. After the 1-month and 6-month follow-ups, no differences were observed between the 2 groups (normal condition of the wound or scar).

On average, each patient was kept in the ICU postoperatively for nearly 24 hours to undergo monitoring. Patients in the HA group spent an average of 10.3 days in the hospital versus 9.7 days for the titanium group (Mann-Whitney test, $\mathrm{p}=0.355$ ). No specific medications were administered to the titanium or HA group (with the exception of second-generation cephalosporin antibiotics for 5 days in the HA group). Accordingly, the hospital costs ought to be nearly the same for both groups.

In this study, it took 10.5 weeks from randomization to implantation in the titanium group and 15 weeks in the HA group (median values), and therefore there was a sig-

TABLE 4. Statistical characteristics of operative procedure and postoperative imaging*

\begin{tabular}{|c|c|c|c|}
\hline Variable & $\mathrm{HA}$ & Titanium & $\mathrm{p}$ Value \\
\hline Mean op time $\pm S D$, mins & $125 \pm 36$ & $102 \pm 36$ & $0.024 \dagger$ \\
\hline Mean duration of anesthesia $\pm S D$, mins & $160 \pm 35$ & $149 \pm 35$ & $0.289 \dagger$ \\
\hline \multicolumn{4}{|l|}{ Intraop complication } \\
\hline Intraop dura opening & $23.10 \%$ & $30.80 \%$ & NS† \\
\hline CSF leakage & $11.50 \%$ & $19.20 \%$ & NS† \\
\hline Intraop AE & 2 & 1 & \\
\hline CT scan quality & & & $<0.001 \ddagger$ \\
\hline w/o artifacts & $21(84)$ & 1 & \\
\hline Low artifacts & 3 & $16(67)$ & \\
\hline Severe restrictions & 1 & 7 & \\
\hline Hematoma on CT & $48 \%$ & $17 \%$ & $0.079 \ddagger$ \\
\hline Subgaleal & 3 & 2 & \\
\hline Epidural & $9(36)$ & 2 & \\
\hline Subdural & 1 & & \\
\hline Epidural hematoma $>1 \mathrm{~cm}$ & $5(20)$ & & $0.03 \S$ \\
\hline Perfect fit of the implant & 19 & 21 & 0.140 \\
\hline Distance $<5 \mathrm{~mm}$ to the bone & 6 & 3 & \\
\hline
\end{tabular}

NS $=$ not significant.

* Values represent the number of patients (\%) unless stated otherwise.

$\dagger$ Mann-Whitney test.

$\ddagger$ Chi-square test.

$\S$ Fisher's exact test. 
TABLE 5. Initial, postoperative, and follow-up mGOS for the HA and titanium groups with no significant differences*

\begin{tabular}{ccccc}
\hline $\begin{array}{c}\text { mGOS } \\
\text { Group }\end{array}$ & Preop & At Discharge & 1-Mo Follow-Up & 6-Mo Follow-Up \\
\hline 1 & $10 / 13$ & $9 / 11$ & $8 / 11$ & $9 / 11$ \\
\hline 2 & $4 / 2$ & $3 / 3$ & $2 / 3$ & $6 / 2$ \\
\hline 3 & $12 / 9$ & $12 / 8$ & $11 / 8$ & $6 / 7$ \\
\hline 4 & \multicolumn{5}{c}{$0 / 2$} \\
\hline 5 & $0 / 1$ & $1 / 1$ & $1 / 1$ & $0 / 2$ \\
\hline * Data are shown as the number of patients in the HA group/number of \\
patients in the titanium group.
\end{tabular}

nificant difference of 4.5 weeks between the 2 groups. According to this parameter, patients in the titanium group participated in the study for 18 weeks before follow-up stopped in comparison with 22 weeks for the HA group (median).

\section{Comparison of Temperature Sensitivity (Heating and Cooling) at} 6 Months After Implantation

Temperature sensitivity was reported by 4 patients in the HA group and 3 patients in the titanium group. It was impossible to differentiate between the scar and the implant itself. However, temperature sensitivity was not permanent and never posed a serious problem for the patients.

\section{Adverse Events}

All in all, 46 AEs were measured in 27 patients (54\%): 13 in the HA group (50\%) and 14 in the titanium group $(58 \%)$. There was no significant difference between the 2 groups. Thirty-eight AEs had a possible causal relationship to implantation. Of the total of 46 AEs, 36 patients recovered completely, 5 patients have not recovered or have sequelae, and 5 patients died. Altogether, 14 SAEs (excluding explantation) were noted (4 patients in the HA group and 3 patients in the titanium group; no significant difference).

\section{Subgroup Analysis}

The operation time for implants smaller than $100 \mathrm{~cm}^{2}$ in both groups was shorter than for implants $>100 \mathrm{~cm}^{2}$. Only 1 patient with an implant $<100 \mathrm{~cm}^{2}$ lost the implant compared with 5 patients with implants $>100 \mathrm{~cm}^{2}$. No significant difference was found between HA and titanium in this subgroup analysis, with the exception of 3 cases of epidural hematomas in the HA group and none in the titanium group.

\section{Discussion}

The aim of this study was to perform a randomized prospective comparison of 2 established cranioplasty materials. Titanium is valued for its stability and biocompatibility, is available in different thicknesses, and can easily be fitted with screws. However, titanium also has disadvantages, such as problems with artifacts on postoperative imaging and the lack of reintegration into the surrounding bone..$^{29}$ Therefore, a leading biocement-HA (CustomBone)-was selected for comparison. Both materials are adapted to the patient's bone defect on the basis of CT data in order to ensure excellent cosmetic accuracy. Furthermore, $\mathrm{HA}$ is osteoconductive and, due to its micro- and macroporosity, its osteointegration has been demonstrated over time in animal experiments as well as single clinical trials. The material shows good compatibility with MRI and CT scanning. Its main disadvantage is the risk of fracture during and immediately after surgery. ${ }^{24}$

Infection rates of $1.77 \%$ have been reported retrospectively in connection with HA in 2887 implants. ${ }^{25}$ In contrast, for custom-made titanium, infection rates of at least $13 \%$ to $16 \%$ for smaller defects ${ }^{8}$ and a marked increase for larger defects have been described. ${ }^{28}$ Therefore, the main aim of the study was to compare the infection rates in both groups.

Comparing the infection rates of local implant-associated wound infection in the first 6 months after operation, the rate of $7.7 \%$ in the HA group was clearly lower than $37.5 \%$ in the titanium group, but without substantial statistical significance. An influence on the rate of reoperation can be assumed since all of those patients underwent reoperation. This outcome might be associated with the prophylactic postoperative antibiotic treatment for 5 days in the HA group demanded by the manufacturer (secondgeneration cephalosporin). In the titanium group, antibiotic treatment was only used in the case of infection.

The number of solely systemic infections was also higher in the titanium group, but again without substantial statistical significance. Considering local and systemic infections together, there is a significant benefit in the HA group (Fisher's exact test, $\mathrm{p}=0.031$ ) (Table 2 ). Due to the lack of significance in the subgroup with local infections, the missing follow-up data for some patients and the potential impact of prophylactic antibiotic treatment in the HA group should be carefully interpreted. Nevertheless, considering the infection rates, there is an advantage for HA. The number of infections observed in the titanium group approximately corresponds to the rate for large defects described in the literature..$^{12}$ Our study mainly included patients with large defects: the average size of the implants was $126.2 \mathrm{~cm}^{2}$ in the titanium group.

On the other hand, the complication rate in our study was about 4 times higher in comparison with the retrospective data for patients with porous HA (CustomBone). The patients included were mainly seriously injured (onethird had a caregiver) with large defects $\left(116.9 \mathrm{~cm}^{2}\right)$, over $80 \%$ showed neurological deficits, and $65 \%$ had preexisting internal diseases. In this study, all infections were documented at the time of occurrence, which cannot be expected from a retrospective postmarketing study. ${ }^{25}$

However, infection was not the only complication parameter. Minor and major complications were observed in $54 \%$ of all patients and as many as $58 \%$ in the titanium group. Since this is the first comprehensive prospective trial, the rates observed approximately match those described in the literature for titanium and HA.

Hill et al. described a retrospective complication rate of $31.5 \%$ for titanium, ${ }^{8}$ while Piedra et al ${ }^{16}$ reported a complication rate of $35 \%$ in smaller defects with bone reconstruction. The reasons for the dramatic differences between the rates observed in the HA group in comparison with the literature have been mentioned previously. ${ }^{25}$ Ultimately, complications occur in 1 of 2 cases. 
Two questions with a vital bearing on everyday life are the reoperation rate and the risk of implant loss, especially in patient information. For the HA group, according to retrospective studies, a $1 \%$ to $2 \%$ rate of reoperation and explantation in $<1 \%$ of cases was indicated. ${ }^{25}$ In this prospective study, $26.9 \%$ of patients in the HA group needed reoperation $(\mathrm{n}=7)$ and $11.5 \%(\mathrm{n}=3)$ needed an explantation (i.e., 1 in 4 patients had to undergo reoperation). In all 3 cases of reoperation, the backup implant was successfully placed. Frassanito et al. reported on a number of children who were younger than 7 years of age with HA. They had a comparable reoperation rate of $20.8 \%$ with implant removal. $^{7}$

There was no significant difference with the titanium group, which had 7 cases of reoperation (29.2\%) and 3 cases of explantation. In 2006, Eufinger et al. showed a comparable reoperation rate of $21 \%$ and secondary implant removal rate in $8.4 \%$ for titanium. ${ }^{6}$ The main reasons for explantation were wound dehiscence with infections in 5 of 6 cases and a single case of infected epidural hematoma. Hill et al. and Mukherjee et al. also reported comparable reoperation rates. ${ }^{8,12}$

Among the reasons for the reoperations in 7 patients in each group, wound dehiscence with infection dominated in the titanium group. The question of postoperative antibiosis may have to be considered, as well when using titanium implants for large defects.

Furthermore, 2 cases of wound infections among the remaining 7 patients were observed in the HA group. In 2 patients, the inserted ventriculoperitoneal shunt systems had to be stopped because of overdrainage syndrome, while the reason for the other 3 reoperations was a spaceoccupying epidural hematoma. Since an HA prosthesis requires extensive preparation of the edge of the dura, the dura is mobilized. This leads to changes in the ratios of CSF and local pressure compared with that before initial shunt insertion. ${ }^{16}$ Accordingly, simultaneous placement of a ventriculoperitoneal shunt system and cranioplasty should be avoided. If a ventriculoperitoneal shunt system is already in place, the patient is preoperatively informed about the risk, and, if possible, adjustable valves are used. ${ }^{13}$

Epidural hematomas evidently occurred frequently in the HA group, and 3 patients needed to undergo reoperation for this purpose. The postoperative CT examination revealed epidural hematomas in 9 cases compared with 2 cases in the titanium group. There is borderline significance according to the chi-square test $(p=0.079)$. One reason for the frequently occurring epidural hematomas may be the small number of predefined holes for dura sutures. According to the manufacturer, more predefined holes could increase the risk of fracture of the implant, although the surgeon may decide to take that risk. It remains to be seen whether this decision can reduce the risk of epidural hematomas. The assessment of the region below the implant is significantly easier in the HA group (chi-square test, $\mathrm{p}<0.001$ ), which was also radiologically confirmed. This should be kept in mind for patients who require frequent follow-up imaging, such as oncology patients. ${ }^{6}$

Surgical duration was significantly longer in the HA group (Mann-Whitney test, $p=0.024$ ), on average 23 minutes longer than in the titanium group. The additional time corresponds to the time needed for the detailed preparation of the edge of the dura and fixation with sutures in the HA group in comparison with fixation with screws in the titanium group. ${ }^{11}$ This additional time could perhaps be shortened by the use of absorbable CranioFix, although it is not officially approved. The duration of anesthesia was not significantly longer, requiring on average an additional 11 minutes in the HA group (Mann-Whitney test, $\mathrm{p}=$ 0.289). The higher occurrence of dura leaks with CSF in the titanium group is probably related to the faster preparation.

Patients with titanium implants and those with HA implants (CustomBone) spent about 24 hours in the ICU postoperatively and another 10 days in the hospital; there were no significant differences, and so the effort and expenditure for the hospital are about the same. Manufacturing of the implant took about 4 weeks during the study, although this has since been reduced to 2 to 3 weeks.

\section{Clinical Symptoms and Neurology}

The level of preexisting internal diseases and neurological deficits were comparable in both groups at the beginning of the study (internal diseases in $>80 \%$ and neurological deficits in $>63 \%$ of patients) and did not change significantly during the study. The only conclusion to be drawn from this is that many severely ill patients participated in the study. This is confirmed by the KPS score, which was on average $70 \%$ in both groups at the beginning of the study (including patients with scores of $20 \%$ or 30\%). The improvement in KPS score at the end of the study (average 77\%) indicates the positive effect of implant treatment on patients, with no significant differences between the 2 groups. Throughout the study, no significant changes in GCS score were measured. , $26,27^{2}$

A more sensitive instrument that was used was the mGOS (an mGOS score of 1 is good). Essentially, patients switched between mGOS Score 1 and mGOS Score 3 during the study with slight emphasis on mGOS score of 1 at the end. There was no evidence of a significant change, although the 5 patients who died were excluded. Moreover, most of the retrospective studies described no significant changes in GOS scores for the patients. Only Chibbaro et al. ${ }^{4}$ reported an improvement of $92 \%$ postoperatively in a small prospective study in 2013.

An internally developed evaluation system was used to compare current neurology with preoperative status and defined 3 options: improved, unchanged, or worse. The majority of patients experienced no change in their neurological deficits during the study. Nine patients $(43 \%)$ showed an improvement with fewer neurological deficits in the HA group compared with 5 patients in the titanium group (26.3\%). Statistical significance was not demonstrated (chi-square test, $p=0.709$ ). The reasons for the positive trend observed might be the extensive preparation with mobilization of the dura, changes in local pressure, and the influences of osteointegration. To clarify this, more patients need to be examined. The positive trend should not be overrated with regard to patient rehabilitation since a positive trend could also mean only minimally improved paresis or an increased flow of speech. Otherwise, a prospective study by Paredes et al. ${ }^{14}$ showed a comparable improvement in $40 \%$ of patients with smaller skull defects. 


\section{Subjective Evaluation of Implantation}

The SF-36 survey was used for this study. This questionnaire, which consisted of 8 sets of criteria for physical and mental assessment, was completed before the intervention as well as at the 1- and 6-month follow-ups. Patients with caregivers were excluded from this evaluation. The assumption that the application of porous HA, which is "closer to real bone" than titanium, has a psychological impact on the rating was not borne out since there were no significant differences between the 2 groups in 11 of the 12 sets of criteria. The total psychological score even indicated a significant advantage for titanium (Mann-Whitney U-test, $\mathrm{p}=0.035$ ). There is much evidence that both materials are well accepted by patients. Accordingly, 6 months after intervention, patient satisfaction had significantly improved in both groups in comparison with the preoperative survey (HA group: $79 \%$ to $94.1 \%$; titanium group: $58 \%$ to $94.1 \%$ ). Staffa et al. ${ }^{23}$ reported nearly $100 \%$ patient satisfaction with the HA prosthesis, while with regard to titanium Eufinger et al. ${ }^{6}$ showed that $90 \%$ of patients would agree to undergo the operation again.

Patients and physicians were asked about their overall impressions as a subjective additional criterion in the follow-up. Since this criterion did not change during the surveys, it was not rated as helpful when assessing treatment.

The accuracy of fit and cosmetic results were rated as very good in both groups, thus contributing to the high level of patient satisfaction with the implant and surgery. Discrete skin irritations at 1 month after surgery were noticeable but were not significant in $20 \%$ of the patients in the HA group. The most likely reason is the more extensive preparation of the periosteum and subcutaneous tissue.

\section{Temperature Sensitivity}

Temperature sensitivity in connection with titanium has been noted in previous articles in up to $50 \%$ of cases. In both groups, 4 patients reported increased temperature sensitivity (average 16\%). However, these indications were related to the wound or scar from the operation, and titanium cannot be assumed to be genuinely disadvantageous in this respect. ${ }^{6}$ In the titanium group, patients also reported a kind of "gurgle" in the first few months.

\section{Error Analysis}

This intention-to-treat study was assisted by the Center for Clinical Trials with regard to the planning, compilation of the data in a database, and statistical analysis. To ensure the high quality of data acquisition, this study was carried out by the study director. Data acquisition and analysis were performed entirely independent of the funding companies. The initial objective of 150 planned patients could not be achieved due to low recruitment at the study centers. After only 53 patients were recruited in 4 years, the decision was made to stop recruitment due to the poor prospect of meeting the objective of 150 patients in a reasonable period of time. This explains the low statistical significance of data analysis, with trends only being observed for higher local infection rates and antibiotic use in the titanium group, as well as improved neurological outcome in the HA group. Therefore, this study is regarded as a preliminary study for future, more detailed investigations.

\section{Conclusions}

There were high complication rates of about $50 \%$ in both groups. In comparison with retrospective studies, the infection rate was substantially lower in the HA group than in the titanium group. Additionally, the neurological outcome in the HA group was inclined to be better. A disadvantage was the significantly greater number of extradural hematomas in the HA group than in the titanium group. In particular, combination with a shunt system needs to be considered as a risk. A high level of acceptance for both materials was observed among all patients. The study emphasizes that cranioplasty is a high-risk intervention.

We recommend HA implants (CustomBone) for younger patients due to osteointegration, patients at higher risk of infection, and patients who require frequent follow-up imaging. Titanium or other alloplastic materials are better suited in connection with highly sunken defects, epilepsy, existing shunt systems, extensive bifrontal defects, and possibly skull base defects.

\section{Acknowledgments}

The authors wish to thank the Clinical Trial Centre Leipzig for its statistical and clinical support.

\section{References}

1. Archavlis E, Carvi Y Nievas M: The impact of timing of cranioplasty in patients with large cranial defects after decompressive hemicraniectomy. Acta Neurochir (Wien) 154:1055-1062, 2012

2. Bender A, Heulin S, Röhrer S, Mehrkens J-H, Heidecke V, Straube A, et al: Early cranioplasty may improve outcome in neurological patients with decompressive craniectomy. Brain Inj 27:1073-1079, 2013

3. Chang V, Hartzfeld P, Langlois M, Mahmood A, Seyfried D: Outcomes of cranial repair after craniectomy. J Neurosurg 112:1120-1124, 2010

4. Chibbaro S, Vallee F, Beccaria K, Poczos P, Makiese O, Fricia M, et al: [The impact of early cranioplasty on cerebral blood flow and its correlation with neurological and cognitive outcome. Prospective multi-centre study on 24 patients.] Rev Neurol (Paris) 169:240-248, 2013 (Fr)

5. De Bonis P, Frassanito P, Mangiola A, Nucci CG, Anile C, Pompucci A: Cranial repair: how complicated is filling a "hole"? J Neurotrauma 29:1071-1076, 2012

6. Eufinger H, Weihe S, Scherer P, Rasche C, Wehmöller M: Management of cranial and craniofacial bone defects with prefabricated individual titanium implants: follow-up and evaluation of 166 patients with 169 titanium implants from 1994-2000. Int J CARS 1:197-203, 2006

7. Frassanito P, Tamburrini G, Massimi L, Di Rocco C, Nataloni A, Fabbri G, et al: Post-marketing surveillance of CustomBone Service implanted in children under 7 years old. Acta Neurochir (Wien) 157:115-121, 2015

8. Hill CS, Luoma AMV, Wilson SR, Kitchen N: Titanium cranioplasty and the prediction of complications. Br J Neurosurg 26:832-837, 2012

9. Huang YH, Lee TC, Yang KY, Liao CC: Is timing of cranioplasty following posttraumatic craniectomy related to neurological outcome? Int J Surg 11:886-890, 2013

10. Kolias AG, Bulters DO, Cowie CJ, Wilson MH, Afshari FT, Helmy A, et al: Proposal for establishment of the UK Cranial Reconstruction Registry (UKCRR). Br J Neurosurg 28:310314,2014

11. Lethaus B, Bloebaum M, Essers B, ter Laak MP, Steiner T, 
Kessler P: Patient-specific implants compared with stored bone grafts for patients with interval cranioplasty. J Craniofac Surg 25:206-209, 2014

12. Mukherjee S, Thakur B, Haq I, Hettige S, Martin AJ: Complications of titanium cranioplasty--a retrospective analysis of 174 patients. Acta Neurochir (Wien) 156:989-998, 2014

13. Oh CH, Park CO, Hyun DK, Park HC, Yoon SH: Comparative study of outcomes between shunting after cranioplasty and in cranioplasty after shunting in large concave flaccid cranial defect with hydrocephalus. J Korean Neurosurg Soc 44:211-216, 2008

14. Paredes I, Castaño-León AM, Munarriz PM, Martnez-Perez R, Cepeda S, Sanz R, et al: Cranioplasty after decompressive craniectomy. a prospective series analyzing complications and clinical improvement. Neurocirugia (Astur) 159:1-9, 2014

15. Piedra MP, Nemecek AN, Ragel BT: Timing of cranioplasty after decompressive craniectomy for trauma. Surg Neurol Int 5:25, 2014

16. Piedra MP, Ragel BT, Dogan A, Coppa ND, Delashaw JB: Timing of cranioplasty after decompressive craniectomy for ischemic or hemorrhagic stroke. J Neurosurg 118:109-114, 2013

17. Piitulainen JM, Kauko T, Aitasalo KMJ, Vuorinen V, Vallittu PK, Posti JP: Outcomes of cranioplasty with synthetic materials and autologous bone grafts. World Neurosurg 83:708-714, 2015

18. Rana OR, Schröder JW, Kühnen JS, Saygili E, Gemein C, Zink MD, et al: The Modified Glasgow Outcome Score for the prediction of outcome in patients after cardiac arrest: a prospective clinical proof of concept study. Clin Res Cardiol 101:533-543, 2012

19. Reddy S, Khalifian S, Flores JM, Bellamy J, Manson PN, Rodriguez ED, et al: Clinical outcomes in cranioplasty: risk factors and choice of reconstructive material. Plast Reconstr Surg 133:864-873, 2014

20. Schuss P, Vatter H, Marquardt G, Imöhl L, Ulrich CT, Seifert V, et al: Cranioplasty after decompressive craniectomy: the effect of timing on postoperative complications. J Neurotrauma 29:1090-1095, 2012

21. Seitz IA, Adler N, Odessey E, Reid RR, Gottlieb LJ: Latissimus dorsi/rib intercostal perforator myo-osseocutaneous free flap reconstruction in composite defects of the scalp: case series and review of literature. J Reconstr Microsurg 25:559-567, 2009

22. Staffa G, Nataloni A, Compagnone C, Servadei F: Custom made cranioplasty prostheses in porous hydroxy-apatite using 3D design techniques: 7 years experience in 25 patients. Acta Neurochir (Wien) 149:161-170, 2007

23. Staffa G, Barbanera A, Faiola A, Fricia M, Limoni P, Mottaran R, et al: Custom made bioceramic implants in complex and large cranial reconstruction: a two-year follow-up. J Craniomaxillofac Surg 40:e65-e70, 2012

24. Stefini R, Esposito G, Zanotti B, Iaccarino C, Fontanella MM, Servadei F: Use of "custom made" porous hydroxyapa- tite implants for cranioplasty: postoperative analysis of complications in 1549 patients. Surg Neurol Int 4:12, 2013

25. Stefini R, Zanotti B, Nataloni A, Martinetti R, Scafuto M, Colasurdo M, et al: The efficacy of custom-made porous hydroxyapatite prostheses for cranioplasty: evaluation of postmarketing data on 2697 patients. J Appl Biomater Funct Mater 13:e136-e144, 2015

26. Stelling H, Graham L, Mitchell P: Does cranioplasty following decompressive craniectomy improve consciousness? Br J Neurosurg 25:407-409, 2011

27. Wachter D, Reineke K, Behm T, Rohde V: Cranioplasty after decompressive hemicraniectomy: underestimated surgery-associated complications? Clin Neurol Neurosurg 115:1293-1297, 2013

28. Wiggins A, Austerberry R, Morrison D, Ho KM, Honeybul $\mathrm{S}$ : Cranioplasty with custom-made titanium plates -14 years experience. Neurosurgery 72:248-256, 2013

29. Williams LR, Fan KF, Bentley RP: Custom-made titanium cranioplasty: early and late complications of 151 cranioplasties and review of the literature. Int J Oral Maxillofac Surg 44:599-608, 2015

30. Zanaty M, Chalouhi N, Starke RM, Clark SW, Bovenzi CD, Saigh M, et al: Complications following cranioplasty: incidence and predictors in 348 cases. J Neurosurg 123:182188,2015

31. Zweckberger K, Juettler E, Bösel J, Unterberg WA: Surgical aspects of decompression craniectomy in malignant stroke: review. Cerebrovasc Dis 38:313-323, 2014

\section{Disclosures}

The authors report the following. Dr. Linder and Ms. SchlothoferSchumann received clinical or research support for the described study from Finceramica and Codman (Johnson \& Johnson $\mathrm{GmbH})$. Finceramica and Codman both provided the funding used for supporting the site management organization and administrative study management, both of which were conducted by the Clinical Trial Centre Leipzig. Influence or bias on the study outcome is excluded.

\section{Author Contributions}

Conception and design: Lindner, Meixensberger. Acquisition of data: Lindner, Schlothofer-Schumann, Kern, Marx. Analysis and interpretation of data: Lindner, Schlothofer-Schumann, Müns. Drafting the article: Lindner, Schlothofer-Schumann. Critically revising the article: Kern, Marx, Müns, Meixensberger. Statistical analysis: Müns. Administrative/technical/material support: Kern, Marx, Meixensberger. Study supervision: Lindner, Meixensberger.

\section{Correspondence}

Dirk Lindner, Department of Neurosurgery, University Hospital Leipzig, Liebigstrasse 20, Leipzig D-04103, Germany. email: dlind@medizin.uni-leipzig.de. 